\title{
Measurements of Aeroacoustic Noise and Pressure Fluctuation Generated by a Door-Mirror Model Placed on a Flat Plate*
}

\author{
Chisachi KATO $^{* *}$ Osamu MURATA ${ }^{* * *}$ Ayumi KOKUBO ${ }^{* * * *}$ \\ Kenichi ICHINOSE $^{* * * * *}$ Takashi KIJIMA ${ }^{* * * * * *}$ Nariaki HORINOUCHI
and Akiyoshi IIDA \\ **Institute of Industrial Science, The University of Tokyo, \\ 4-6-1 Komaba, Meguro-ku, Tokyo 153-8505, Japan \\ E-mail: ckato@iis.u-tokyo.ac.jp \\ ***Toyota Central R\&D Laboratories, \\ 41-1 Yokomichi, Nagakute, Nagakute-cho, Aichi-gun, Aichi 480-1192, Japan \\ ****Hitachi Home \& Life Solutions, Inc., \\ 800 Tomida, Oohira-machi, Shimotsuga-gun, Tochigi, 329-4493, Japan

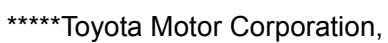 \\ 1 Toyota-Cho, Toyota City, Aichi 471-8571, Japan \\ ******Ichikoh Industries, Ltd., \\ 80 Itado, Isehara-shi, Kanagawa-ken 259-1192 \\ *******Kogakuin University \\ 2665-1 Nakano, Hachioji, Tokyo 192-0015, Japan
}

\begin{abstract}
To provide reliable validation data for computational aeroacoustics methods that predict bluff-body sound, the far-field sound pressure and fluctuating surface pressure were measured in detail for a simplified mock-up model of an automobile door mirror (a quarter-section sphere mounted on a half-circular cylinder placed on a flat plate). The surface flow on the model was visualized using an oil flow method. The Reynolds number based on the upstream uniform wind velocity and the diameter of the half-circular cylinder was varied from $1.4 \times 10^{5}$ to $2.4 \times 10^{5}$ for model yaw angles of 0 and 15 degrees. The pressure fluctuations on the model surface and flat plate indicated the existence of large-scale vortical motion with a non-dimensional frequency, calculated on the basis of the model width and the free-stream velocity, of 0.16 . The frequency spectra of the sound pressure fluctuations had a weak peak apparently due to the large-scale vortical motion. However, unlike the flow around a two-dimensional circular cylinder, the peak was not intense enough to alter the overall level of the sound radiated from the model. When the model was rotated 15 degrees, the power spectra of the sound pressure increased in the non-dimensional frequency range between 1 and 2 .
\end{abstract}

Key words: Aerodynamic Noise, Door Mirror, Pressure Fluctuation, Measurement, Validation

\footnotetext{
*Received 22 Dec., 2006 (No. T-04-1290) Japanese Original : Trans. Jpn. Soc. Mech. Eng., Vol.71, No.710, B (2005), pp.2471-2479 (Received 7 Dec, 2004) [DOI: 10.1299/jee.2.278]
}

\section{Introduction}

As other automobile noise sources, such as the engine and tires, have become less of a 
problem, aerodynamic noise has become the dominant noise source under high-speed cruising conditions. Among the various types of aerodynamic noise, the broadband noise that is generated by air flowing around the front pillars and door mirrors is probably the most noticeable. It is caused by fluctuations in the static pressure of the complex flow field, and its generation or propagation mechanism has not yet been fully clarified. While numerical simulation has been used to predict this complex flow field and resulting sound field ${ }^{(1)-(6)}$, quantitative prediction of the frequency spectra of the sound pressure level has not yet been achieved. Moreover, reliable and detailed experimental data that can be used to verify the accuracy of such a prediction are not found in the literature.

To obtain reliable experimental data for use in such validation, we measured the flow field and resulting sound field around a simplified mock-up model of a door mirror mounted on a flat plate and placed in a low-noise-level wind tunnel. We also investigated the relationship between the source pressure fluctuations in the flow field and the resulting sound field.

In this paper, we first clarify the location of the separation and transition of the boundary layer that develops on the model surface and describe the structure of the flow around the model based on the distributions of the time-averaged and fluctuating static pressures on the model surface and flat plate. The surface streamlines visualized using an oil film method are also presented. We then discuss the dependency of the sound pressure spectra on the Reynolds number of the flow and directivity of the sound field in relation to the source flow field.

\section{Experimental Apparatus and Measurement Method}

\section{$2 \cdot 1$ Test Model and Wind Tunnel}

Figure 1 shows the shape and dimensions of the test model, a simplified mock-up model of an actual door mirror. A quarter section of a sphere was placed on top of a half cylinder, as in the wind-tunnel test done by Hold ${ }^{(4)}$. The model was mounted on a flat plate that was smoothly connected to the exit nozzle of a wind tunnel.

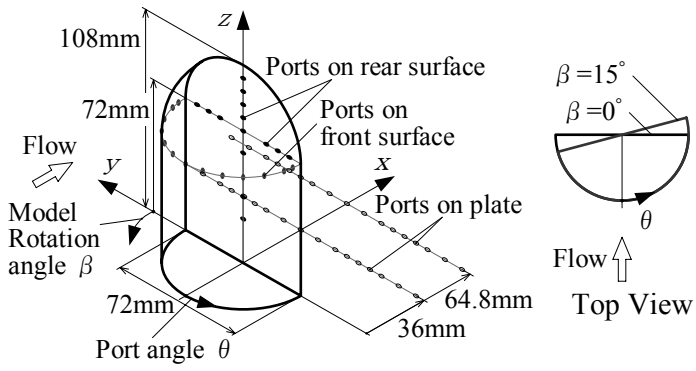

Fig. 1 Test model and static pressure ports

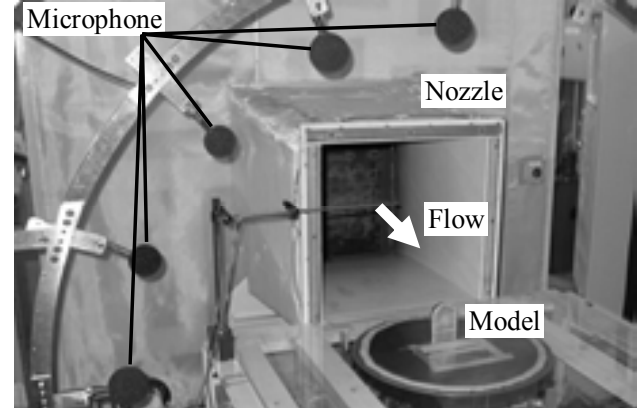

Fig. 2 Experimental apparatus 
The flow around the test model was characterized by large-scale Karman vortices shed from the half cylinder that mixed with the downwash from the top part of the model. This flow should reproduce the important features of the complex flow field around an actual automobile door mirror.

The model was made of aluminum by mechanical cutting; the trailing edge of the model was rounded with a margin of $0.1 \mathrm{~mm}$. The diameter, $D$, of the half cylinder was 72 $\mathrm{mm}$, and the height, $H$, of the model was $108 \mathrm{~mm}$. The ratio of model blockage to the wind tunnel cross-section was $2.9 \%$.

We defined Cartesian coordinates for later reference using $x, y$, and $z$ axes aligned with the main flow, the lateral flow, and the vertical flow, respectively. The origin of the coordinates was set at the point where the center line on the rear surface of the model intersected the flat plate.

All the measurements were made in a low-noise-level low-speed wind tunnel at the Institute of Industrial Science, The University of Tokyo. It is a semi-open Göttingen type. The open test section is $500 \times 500 \mathrm{~mm}$ (cross-section) $\times 1760 \mathrm{~mm}$ (streamwise) and is installed in an anechoic chamber $(4200 \mathrm{~W}, 1900 \mathrm{H}$, and $5000 \mathrm{~L} \mathrm{~mm})$. At a wind speed of 40 $\mathrm{m} / \mathrm{s}$, the non uniformity and turbulence intensity of the main flow are under 1.0 and $0.5 \%$, respectively, and the background noise level is $56.9 \mathrm{~dB}$ A-weighted.

The test model was placed $250 \mathrm{~mm}$ downstream of the nozzle exit on a flat plate, as shown in figure 2 , where the thickness of the boundary layer that developed on the flat plate was estimated to be $6 \mathrm{~mm}$.

We measured the time-averaged and fluctuating static pressures on the surfaces of the test model as well as on the flat plate by using small static pressure sensors (Endevco $8507 \mathrm{C}-2$, range: $14 \mathrm{kPa}$, frequency: $70 \mathrm{kHz}$ ). They were installed as shown in figure 3 . They sensed the static pressure of the air flow around the test model through small holes (diameter: $0.5 \mathrm{~mm}$, length: $1.5 \mathrm{~mm}$ ). Their frequency characteristics are described below.

To investigate the directivity of the sound that radiated from the test model, we measured the noise with non-directivity microphones (RION UC-31, NH-04) placed at 45 points outside the air flow, as shown in figure 4.

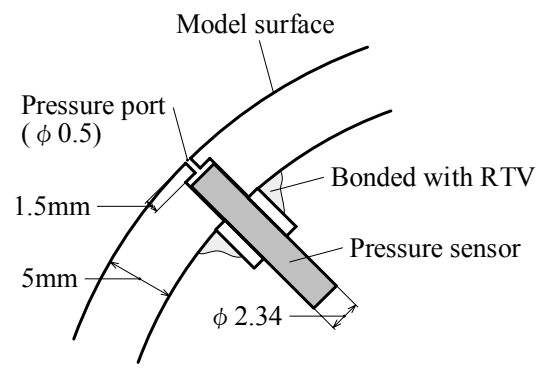

Fig. 3 Mounted static-pressure sensor

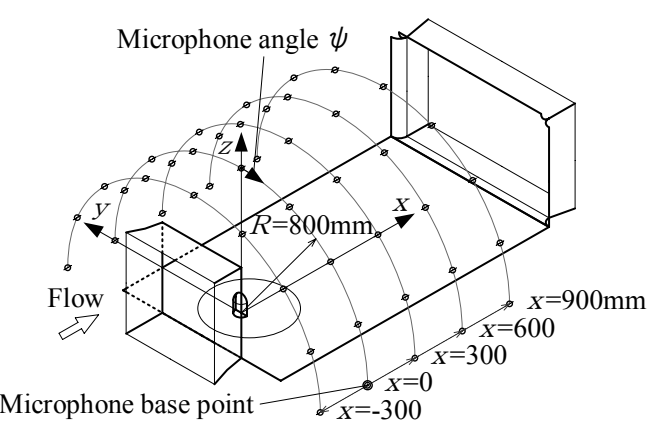

Fig. 4 Experimental set-up and microphone positions 


\section{$2 \cdot 2$ Uncertainty of Measured Data}

The estimated Helmholtz resonance frequency of the static pressure sensors is given by

$$
f_{\text {res }}=\frac{c}{2 \pi} \cdot \sqrt{\frac{A}{l \cdot V}} \doteqdot 8.3 \mathrm{kHz}
$$

where

$c$ : speed of sound $(340 \mathrm{~m} / \mathrm{s})$

$A$ : throat area $\left(2.0 \times 10^{-7} \mathrm{~m}^{2}\right)$

$l$ : throat length $\left(1.5 \times 10^{-3} \mathrm{~m}\right)$

$V$ : cavity volume $\left(5.7 \times 10^{-9} \mathrm{~m}^{3}\right)$.

Preliminary testing using a speaker showed that the actual resonance frequency was 7.3 $\mathrm{kHz}$, which agrees reasonably well with the estimation above. We thus considered the frequency spectra of static pressure fluctuations over $7.0 \mathrm{kHz}$ to be contaminated by the resonance effects of the sensors and discarded them during data processing.

Although the anechoic chamber prevented undesired reflections of the radiated sound at the chamber wall, the acoustical field in the chamber is not a perfect free space-it is somewhat affected by the chamber. This is most noticeable for low-frequency sounds. In our measurements, the sound pressure spectra lower than $70 \mathrm{~Hz}$ were affected (as shown in figure 15) by the acoustical field within the chamber and thus discarded during data processing.

\section{3 Data Logging and Post Processing}

The fluctuations in surface static pressure and sound pressure were logged into a personal computer through a data logger (Keyence NR-2000) after their signals were amplified by a strain amplifier (TEAC SA-59 for surface pressure and RION UN-04 for sound pressure).

The data sampling rate was $40 \mathrm{kHz}$, and a total of 550,000 samples was obtained for each of the sampled data during the sampling period of $13.75 \mathrm{~s}$. The frequency spectra of the pressure fluctuations were obtained by performing fast Fourier transform on each of the 16,384 sampled data after applying the Hanning window function to the raw data. As a result, the computed frequency spectra have a resolution of $2.44 \mathrm{~Hz}$ and a maximum frequency of $20 \mathrm{kHz}$.

The pressure was normalized using the dynamic pressure of the free stream.

$$
C p \equiv \frac{p-p_{\mathrm{a}}}{\left(\rho U_{S}{ }^{2}\right) / 2},
$$

where

$C_{p}$ : static pressure coefficient

$p$ : static pressure measured at pressure holes

$p_{\mathrm{a}}$ : static pressure of free stream

$\rho$ : fluid density

$U_{S}$ : free-stream velocity.

We defined the normalized static pressure fluctuation, $C_{p}{ }^{\text {}}$, in the same way as $C_{p}$.

The sound pressure levels were calculated by referring to the minimum audible pressure of $P_{0}\left(=2.0 \times 10^{-5} \mathrm{~Pa}\right)$, and the $1 / 1$ octave-band sound pressure levels were obtained where needed. 
The measured frequency, $f_{\mathrm{S}}$, was normalized using free-stream velocity $U_{\mathrm{S}}$ and diameter $D$ of the model:

$$
\mathrm{St} \equiv f_{\mathrm{s}} \cdot \frac{D}{U_{\mathrm{s}}}
$$

To ascertain the similarity of the frequency spectra, we used a converted frequency $f$ corresponding to reference free-stream velocity $U(40 \mathrm{~m} / \mathrm{s})$ :

$$
f=f_{\mathrm{s}} \cdot \frac{U}{U_{\mathrm{s}}}
$$

\section{2・ 4 Experimental Conditions}

Free-stream velocity $U_{\mathrm{S}}$ was set to 30,40 , or $50 \mathrm{~m} / \mathrm{s}$, resulting, respectively, in a Reynolds number, $R e$, of $1.4,1.9$, or $2.4 \times 10^{5}$ based on diameter $D$ of the model. The $40 \mathrm{~m} / \mathrm{s}$ case $\left(R e\right.$ of $\left.1.9 \times 10^{5}\right)$ was regarded as the reference case.

The Reynolds numbers above are below the critical Reynolds number for both a circular cylinder $\left(R e_{\mathrm{c}} \cong 5 \times 10^{5}\right)$ and a sphere $\left(R e_{\mathrm{c}} \cong 3 \times 10^{5}\right)$ above which the flow transitions to turbulence in the boundary layer before it separates from the surface. Thus, the boundary layer on the test model was presumed to separate from the model surface while it was still laminar. This was later confirmed by the distributions of the measured static pressure on the model surface.

The model was rotated from 0 to 15 degrees; i.e., the yaw angle, $\beta$, was set to 0 or 15 degrees, as shown in figure 1 . For both cases, we plotted the static pressure on the test model against the asimuzal angle, $\theta$, with its origin (zero) set to the point where the time-averaged pressure was maximum.

\section{Results and Discussion}

\section{3·1 Surface Pressure Distributions}

\section{$3 \cdot 1 \cdot 1$ Front Surface}

Figure 5 shows the time-averaged and fluctuating static pressure distributions on the front surface of the model for a model yaw angle of zero $(\beta=0)$. The measurements were taken at the intersection between the quarter-section sphere and the half cylinder $(z / D=1.0)$. Time-averaged distributions around a circular cylinder ${ }^{(8)}$ and a sphere ${ }^{(9)}$ for a sub-critical Reynolds number are also shown for reference. The measured static pressure had a symmetric distribution around $\theta=0$, as expected. The value of $C_{P}$ was a little less than 1.0 at $\theta=0$, meaning that the mean flow was not stagnant at this point. There was a weak upward flow. The time-averaged distributions for the test model are between those for the circular cylinder and sphere and have a minimum at around $\theta= \pm 60^{\circ}$. This minimum is due to the separation of the boundary layer from the model surface and indicates that the separation occurred a little earlier (upstream) than for the circular cylinder and sphere.

Visualization of the surface streamlines using an oil flow method (figure 6) revealed that the boundary layer separated from the model surface at around $70^{\circ}$. The static pressure fluctuations shown in figure 5 had maximums at around $\theta= \pm 75^{\circ}$, immediately downstream of the separation point. The distributions of the time-averaged and fluctuating static pressures on the front surface of the model did not reveal any noticeable dependency on the Reynolds number of the flow. 
The time-averaged and fluctuating static pressures on the front side of the model were compared for model yaw angles of 0 and 15 degrees. As shown in figure 7, the time-averaged pressure coefficient distributions were almost identical. For an angle of 15 degrees, the pressure on the suction surface $(\theta>0)$ was almost constant downstream of the separation point (around $70^{\circ}$ ), indicating that no reattachment occurred downstream of the separation point. The fluctuating pressure distributions, in contrast, showed small differences. The values were slightly higher for an angle of 15 degrees, presumably due to enhanced fluctuations in the wake. (This will be shown later in figure 13.)

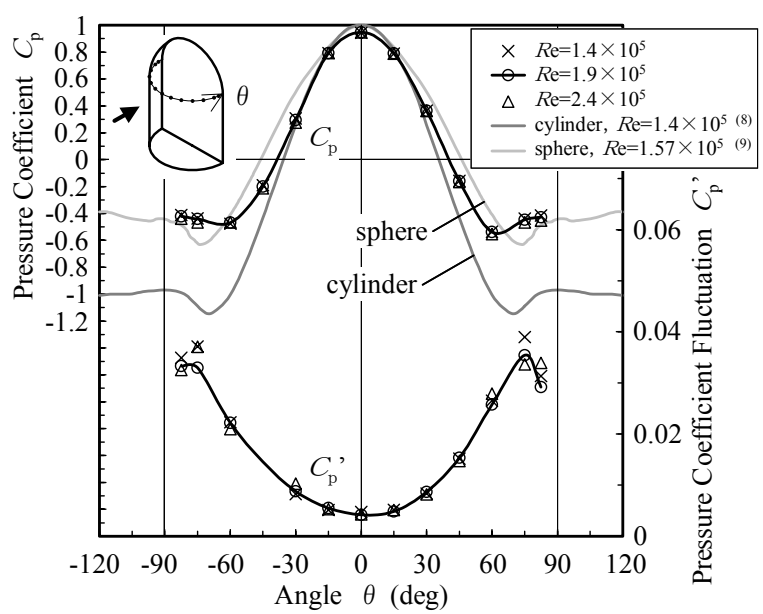

Fig. 5 Distribution of static pressure coefficients and their fluctuation on front surface $\left(z / D=1.0, \beta=0^{\circ}\right)$

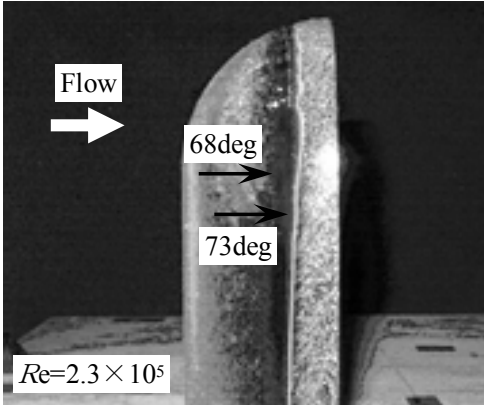

Fig. 6 Oil flow pattern on front surface

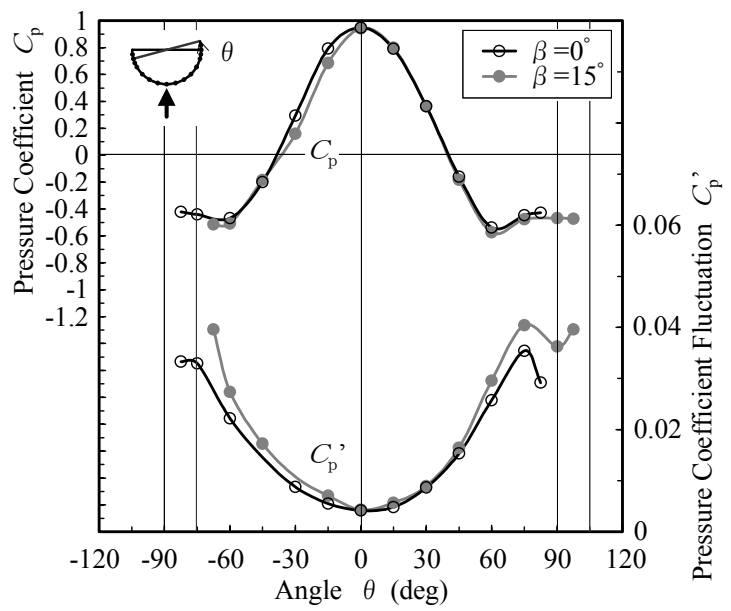

Fig. 7 Distribution of static pressure coefficients and their fluctuation on front surface $(z / D=1.0, R \mathrm{e}=$ $\left.1.9 \times 10^{5}\right)$ 
Figure 8 (a) shows the frequency spectra of the static pressure fluctuations at $60^{\circ}$ from the leading edge for yaw angles of 0 and 15 degrees. Both spectra show a weak peak at around a Strouhal number, St, of $0.16(f=90 \mathrm{~Hz})$. This peak was apparently due to large-scale, periodic shedding of vortices from the test model. They were similar to Karmann vortices but with a frequency slightly lower than that for the usual Karmann vortices $\quad(S t=0.18-0.2)$ for this Reynolds number range. This presumably resulted from the smaller separation velocity for this case, as shown in figure 5. Comparison of the frequency spectra for yaw angles of 0 and 15 degrees revealed slightly higher values for the zero-degree case in the $S t$ frequency range of 0.7 to 2.7 ( $f=500 \mathrm{~Hz}-1.5 \mathrm{kHz})$. This will be discussed in detail later in conjunction with the sound pressure level (subsection 3.2).

Figure 8 (b) shows the frequency spectra of the static pressure fluctuations at several points on the front side of the model surface for a yaw angle of 15 degrees. For $\theta=90.0$ and $97.5^{\circ}$, fluctuations in the static pressure rapidly increased for $S t=1-10(f=560 \mathrm{~Hz}-5.6$ $\mathrm{kHz}$ ), apparently associated with boundary layer separation and subsequent transition of the flow to turbulentce. However, even at $\theta=97.5^{\circ}$, the separated flow did not seem to have become fully turbulent, which can be confirmed by comparing its frequency spectra with those at the rear surface of the model (see figure 11).

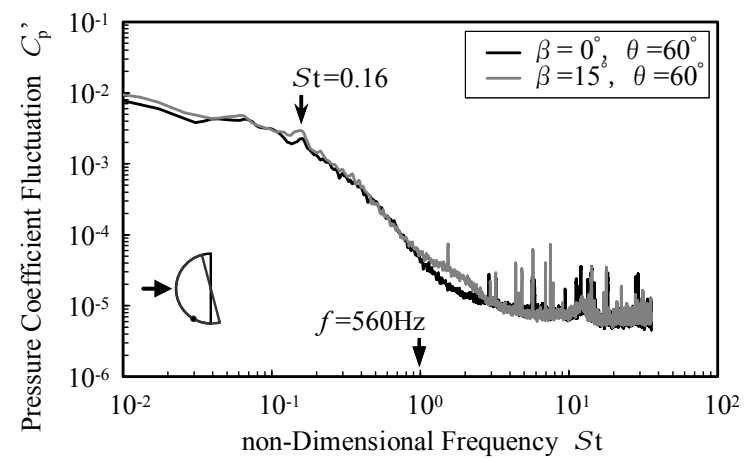

(a) $\theta=60$

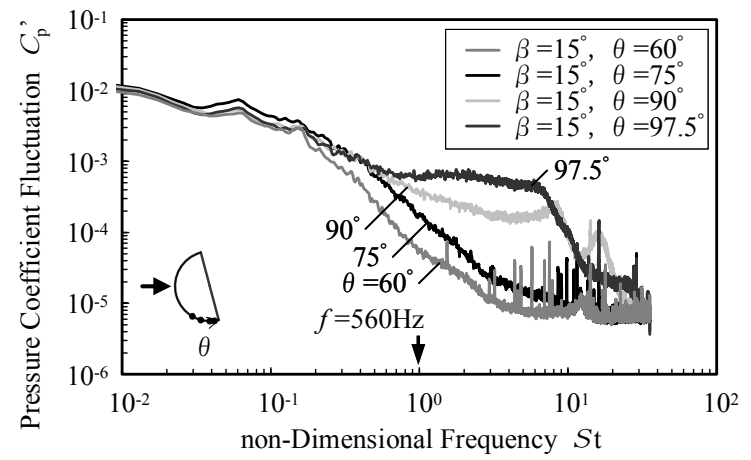

(b) $\theta=60 \sim 97.5$

Fig. 8 Power spectra of pressure coefficient fluctuation in front surface $\left(z / D=1.0, R e=1.9 \times 10^{5}\right)$

\section{3·1 2 Rear Surface}

Figure 9 shows the distributions of the time-averaged and fluctuating static pressures at the rear surface of the model along horizontal line $\mathrm{z} / D=1.0$ for a yaw angle of zero. The time-averaged pressure had little dependency on the Reynolds number while the level of the fluctuations slightly increased with the Reynolds number. The separation velocities were almost the same for the three Reynolds numbers, as shown in figure 5. Therefore, the 
transition and/or formation of large-scale vortices presumably took place closer to the rear surface of the model the higher the Reynolds number. This caused enhanced mixing (fluctuation) on the rear side of the model.

Figure 10 shows the distributions of the time-averaged and fluctuating static pressures at the rear surface of the model along vertical line $y / D=0.0$ for a yaw angle of zero. Similar to the distributions along horizontal line $\mathrm{z} / D=1.0$, the time-averaged pressure had little dependency on the Reynolds number while the level of fluctuations slightly increased with the Reynolds number. The time-averaged and fluctuating pressures, respectively, had a local maximum and minimum at around $z / \mathrm{D}=1.1$, which indicates that this is the rear stagnation point. We verified that these local maximum and minimum were not caused by the individual characteristics of the pressure sensors. Near the bottom wall $(z / D=0.0)$, the fluctuations rapidly increased, probably due to the horse vortices that formed at the corner between the model surface and bottom wall. The pressure distributions for a yaw angle of 15 degrees (not shown here) were similar.

The frequency spectra of the static pressure fluctuations are plotted in figure 11 for several points along vertical line $y / D=0.0$ on the rear surface of the model. Each spectrum closely follows the $-5 / 3$ power law of turbulence in the $S t$ range 0.1 to 10.0 ; their power suddenly dropped due to the characteristics of the measurement system, upper-bound by the occurrence of Helmholtz resonance. This indicates that the flow on the rear surface of the model was in a fully developed turbulent state. Note that the weak peak at around an St of 0.16 on the front surface of the model was not seen on the rear surface.

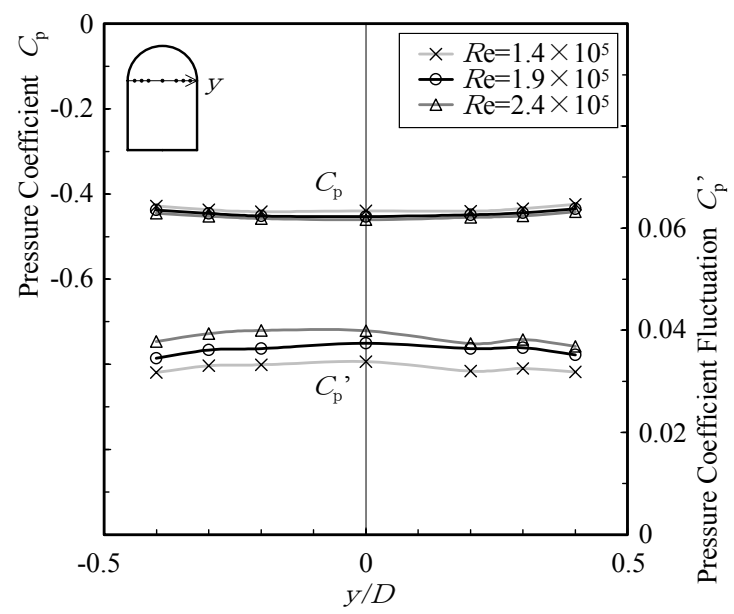

Fig. 9 Distribution of static pressure coefficients and their fluctuation along horizontal line on rear surface $\left(z / D=1.0, \beta=0^{\circ}\right)$ 


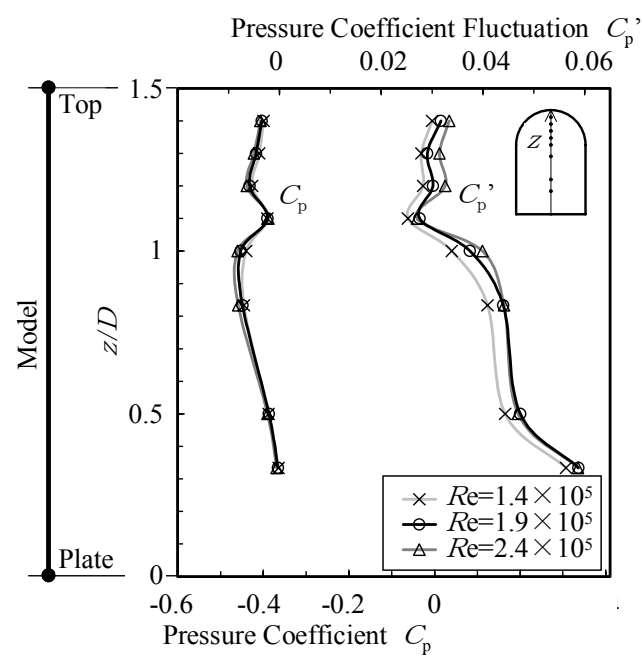

Fig. 10 Distribution of static pressure coefficients and their fluctuation along vertical line on rear surface $\left(y / D=0.0, \beta=0^{\circ}\right)$

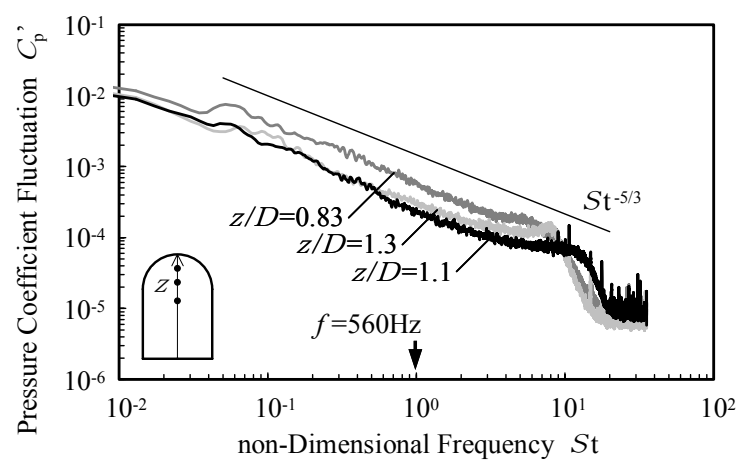

Fig. 11 Power spectra of pressure coefficient fluctuation in rear surface $\left(y / D=0.0, \beta=0^{\circ}, R \mathrm{e}=1.9 \times 10^{5}\right)$

\section{$3 \cdot 1 \cdot 3$ End Wall Surface}

Figure 12 shows the distributions of the time-averaged and fluctuating static pressure at $x / D=0.5$ and $x / D=0.9$ on the flat plate for a yaw angel of zero. The time-averaged distributions show the spread range of the wake of the model while the peak positions in the fluctuating distributions indicate the location of the shear layer in the wake. The wake widened from $x / D=0.5$ to 0.9 . Note that the peak value of the fluctuating static pressure, $C_{p}$, was 0.13 for $x / D=0.5$ and 0.19 for $x / D=0.9$, which implies that the wake of the model was still growing in this region. These peak values are several times greater than those for the rear surface of the model (0.03-0.04), as shown in figure 9. This clearly shows that large-scale vortex shedding took place behind the model. (Figure 14 shows the frequency spectra of the static pressure fluctuations at the point where they took a maximum value. The peak at around $S t=0.16$ confirms the formation of large-scale vortices behind the model.) 


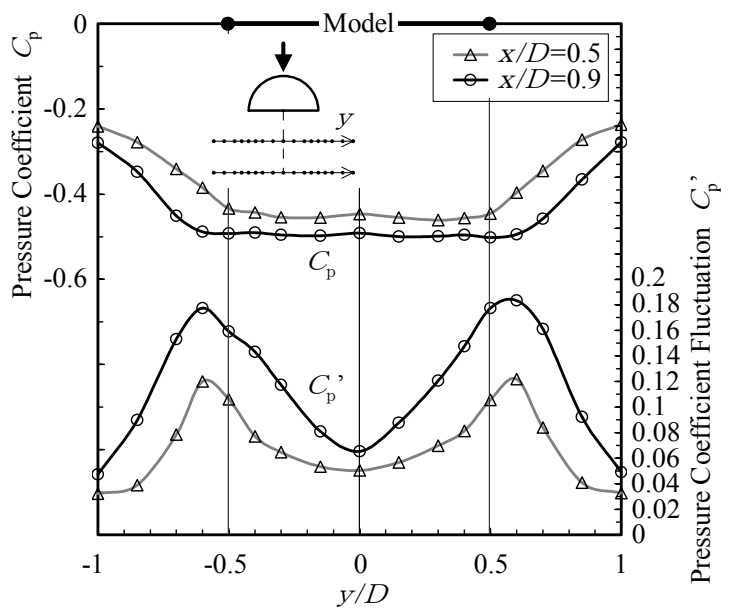

Fig. 12 Distribution of static pressure coefficients and their fluctuation on flat plate $\left(\beta=0^{\circ}, R \mathrm{e}=1.9 \times 10^{5}\right)$

Figure 13 shows, for model yaw angles of 0 and 15 degrees, the time-averaged and fluctuating static pressure distributions along the $x / D=0.9$ line on the flat plate. The 15-degree case showed slightly lower values for the time-averaged static pressure on the suction side $(y / D=-0.6$ to -0.1$)$ and considerably higher peak values for the fluctuating static pressure, both on the suction and pressure sides. The lateral positions of these peaks were shifted slightly toward the positive $y$-direction, indicating that the model turned the main flow around it when the yaw angle was 15 degrees.

Figure 14 shows the frequency spectra of the static pressure fluctuations at the point where these fluctuations took their maximum values for model yaw angles of 0 and 15 degrees. The peak at $S t=0.16$ is presumably the result of the large-scale vortical motion behind the model, as mentioned in the previous subsection. The power spectra at frequencies higher than this peak obeyed the $-5 / 3$ power law of turbulence up to the cut-off frequency, which was determined by the resonance of the measurement system. As already noted in the discussion of figure 12, this power level on the flat plate is approximately one order of magnitude higher than that on the rear surface of the model.

\section{$3 \cdot 2$ Noise Characteristics}

Figure 15 shows the frequency spectra of the sound pressure fluctuations at the reference point shown in figure 4 together with that of the background noise. The noise measured at the reference point was not affected by the noise generated by pressure fluctuations at or reflected on the flat plate, so it can be regarded as the pure noise radiated from the model that directly reached the measurement point. For frequencies higher than 70 $\mathrm{Hz}(S t=0.13)$, the sound pressure level of the test model was appreciably different from that of the background noise, and hence it is regarded as reliable. The weak peak at $90 \mathrm{~Hz}$ $(S t=0.16)$ corresponds to the one in the frequency spectra of the static pressure fluctuations and is attributed to the large-scale vortical motion already mentioned. This peak was not intense enough to alter the overall noise level. Except for the peak at $S t=0.16$, no appreciable peaks were found in the sound pressure spectra, which means that the aerodynamic sound radiated from the test model had characteristics similar to those of broadband noise. To confirm the accuracy of the measured sound pressure spectra, we also measured the sound pressure fluctuations for another model with the identical shape in a different wind tunnel and obtained virtually the same results. Therefore, the sound pressure spectra presented here can be regarded as reliable. 


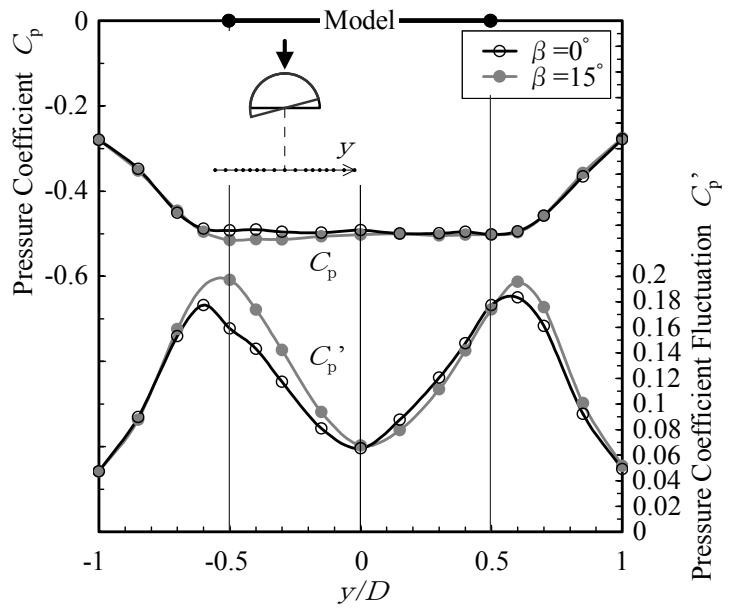

Fig. 13 Distribution of static pressure coefficients and their fluctuation on flat plate $(x / D=0.9, R \mathrm{e}=$ $\left.1.9 \times 10^{5}\right)$

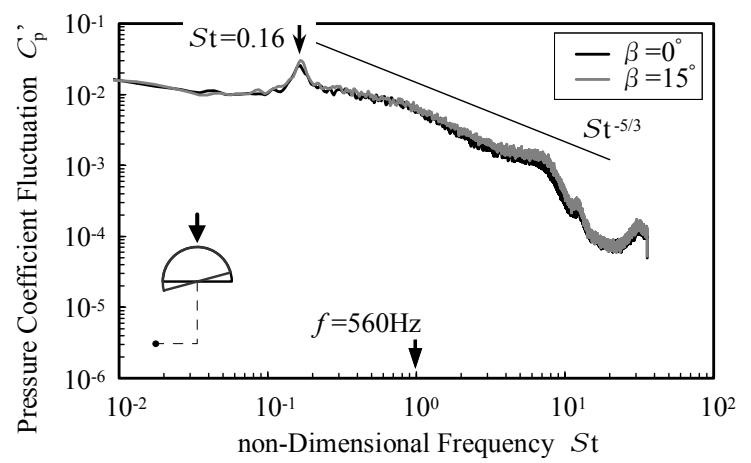

Fig. 14 Power spectra of pressure coefficient fluctuation on flat plate $(x / D=0.9, y / D=-0.6, \operatorname{Re}=$ $\left.1.9 \times 10^{5}\right)$

To investigate the similarity of the measured frequency spectra of the sound pressure fluctuations for different Reynolds numbers, we converted the spectra to values corresponding to the reference free-stream velocity of $40 \mathrm{~m} / \mathrm{s}$ using the following formula. The results are presented in figure 16. We assume that the frequency and sound pressure level are respectively proportional to the $1^{\text {st }}$ and $6^{\text {th }}$ powers of the free stream velocity.

$$
L \mathrm{p}=L \mathrm{p}_{\mathrm{s}}+10 \cdot \log \left(\frac{U}{U_{\mathrm{s}}}\right)^{6}
$$

where $L \mathrm{p}_{\mathrm{s}}$ and $U_{s}$, respectively, denote the measured sound pressure level and free-stream velocity at which the measurements were taken, while $U$ and $L \mathrm{p}$, respectively, denote the reference velocity $(40 \mathrm{~m} / \mathrm{s})$ and sound pressure level converted to the reference velocity. The measured sound pressure spectra closely obeyed the above-mentioned similarity law of the dipole sound at frequencies higher than $150 \mathrm{~Hz}(S t=0.27)$. If we confine our discussion of free-stream velocities to 40 and $50 \mathrm{~m} / \mathrm{s}$, similarity holds even for frequencies down to $120 \mathrm{~Hz}(S t=0.22)$, which seems reasonable because the source fluctuations in the static pressure on the model surface were similar for different Reynolds numbers, as shown in figure 5 .

Figure 17 shows, for model yaw angles of 0 and 15 degrees, the frequency spectra of the sound pressure fluctuations at the point where the difference between the two cases was 
most noticeable. For a frequency range of $400 \mathrm{~Hz}$ to $1.6 \mathrm{kHz}(S t=0.7-2.9)$, the sound pressure level was higher for the 15-degree case, while for 2.2 to $8 \mathrm{kHz}(S t=4-14)$, the opposite was true. As will be discussed later, these differences cannot be attributed to simple rotation of the sound field resulting from model rotation. The model rotation altered the separation of the boundary layer and resulting pressure fluctuations on the model surface.

Figure 18 shows the dependency of the sound pressure levels on the frequency for the three Reynolds numbers at the same point as in figure 17 for a model yaw angle of 15 degrees. A local maximum was observed in the frequency range of $400 \mathrm{~Hz}$ to $1.6 \mathrm{kHz}$, and it slightly shifted toward a lower frequency as the Reynolds number increased. The spectra are not as similar as those for an angle of zero (figure 16).

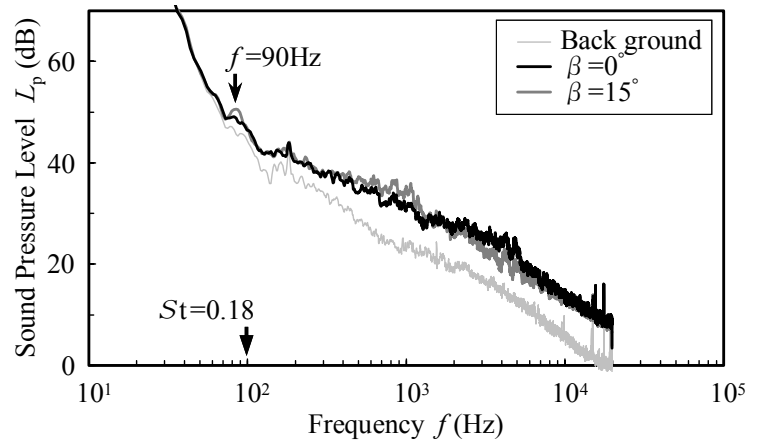

Fig. 15 Power spectra of sound pressure level $\left(x / D=0.0, \psi=90^{\circ}, R \mathrm{e}=1.9 \times 10^{5}\right)$

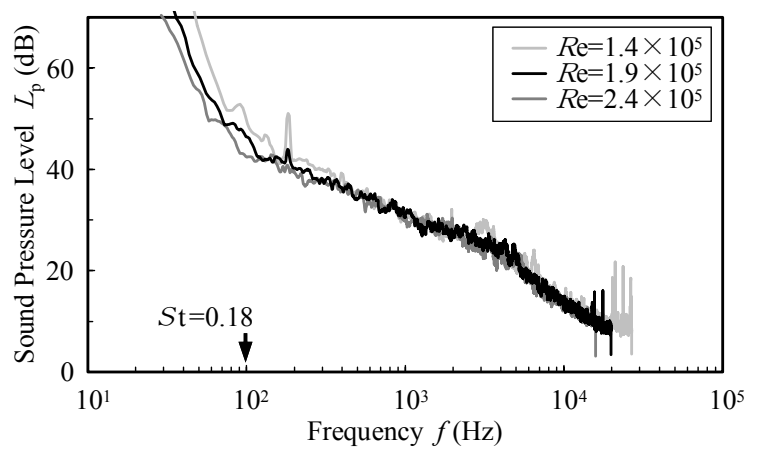

Fig. 16 Similarity of power spectra of sound pressure level for model yaw angle of zero $(x / D=0.0, \psi=$ $90^{\circ}$, reduced to $40 \mathrm{~m} / \mathrm{s}$ )

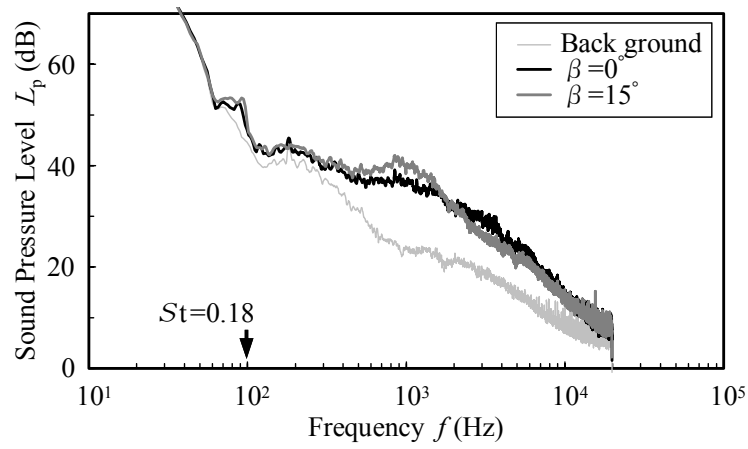

Fig. 17 Effects of model yaw angle on power spectra of sound pressure level $\left(x / D=-4.2, \psi=67.5^{\circ}, R \mathrm{e}=\right.$ $\left.1.9 \times 10^{5}\right)$ 


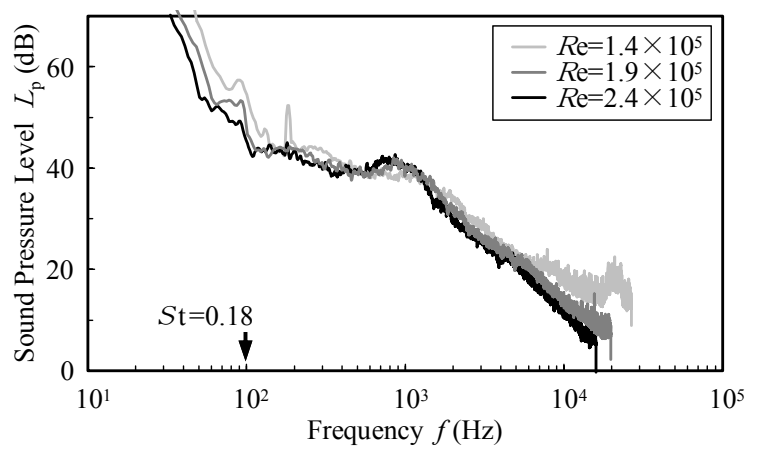

Fig. 18 Similarity of power spectra of sound pressure level for model yaw angle of $15^{\circ}(x / D=-4.2, \psi=$ $67.5^{\circ}$, reduced to $40 \mathrm{~m} / \mathrm{s}$ )

Finally, figure 19 shows, for model yaw angles of 0 and 15 degrees, the spatial distributions of the sound pressure level for two octave-band frequencies centered at $f_{\mathrm{m}}=4$ and $1 \mathrm{kHz}$. We selected these two frequency bands because, at the former frequency, the sound pressure level is higher with a model yaw angle of zero while at the latter it is higher with a model yaw angle of 15 degrees, as shown in figure 17. To take the effects of the distance between the model and measurement point into account, we converted the plotted sound pressure level for each frequency band into a reference distance value using the following formula. Note that the sound generated and/or reflected on the flat plate also contributes to the sound pressure level, except for the $\psi= \pm 90$ degree points.

$$
L \mathrm{p}=L \mathrm{p}_{\mathrm{s}}+10 \cdot \log \left(\frac{R_{\mathrm{s}}}{R}\right)^{2},
$$

where $L \mathrm{p}_{\mathrm{s}}$ and $R_{\mathrm{s}}$, respectively, are the measured sound pressure level and distance at the point where the sound was measured, while $L_{\mathrm{p}}$ is the sound pressure level converted using a reference distance, $R$, of $800 \mathrm{~mm}$.

For the center frequency of $4 \mathrm{kHz}$ shown in figure 19 (a), the sound pressure level showed a nearly symmetric distribution for the center line of the main flow $(y=0)$ for a yaw angle of zero. In this case, the sound pressure was highest at $\psi= \pm 67.5$ degrees. However, this does not necessarily mean that the most intense sound radiated in that direction because, as noted above, the sound that is generated and/or reflected on the flat plate also contributes to the sound pressure level at this measurement point. For a yaw angle of 15 degrees, despite the model rotation, the distribution of the sound pressure level was also nearly symmetric for the centerline of the main flow. The sound pressure level was about $2 \mathrm{~dB}$ higher for the zero-degree case.

For the center frequency of $1 \mathrm{kHz}$ shown in figure 19 (b), the sound pressure level showed a nearly symmetric distribution for center line $y=0$ for a yaw angle of zero, as in figure 19 (a). However, for a yaw angle of 15 degrees, it showed an asymmetric distribution. The sound pressure level was about $2 \mathrm{~dB}$ higher for the 15-degree case in the bottom left and top right regions. As shown in figure 8 (a), for a frequency range of $500 \mathrm{~Hz}$ to $1.5 \mathrm{kHz}$ ( $S t=0.7-2.7)$ the static pressure fluctuations on the model surface were about 3 $\mathrm{dB}$ higher for the 15-degree case, which would account for the higher sound pressure fluctuations in this frequency range. Detailed investigations of the relationship between the flow field and resulting sound field are currently underway. 


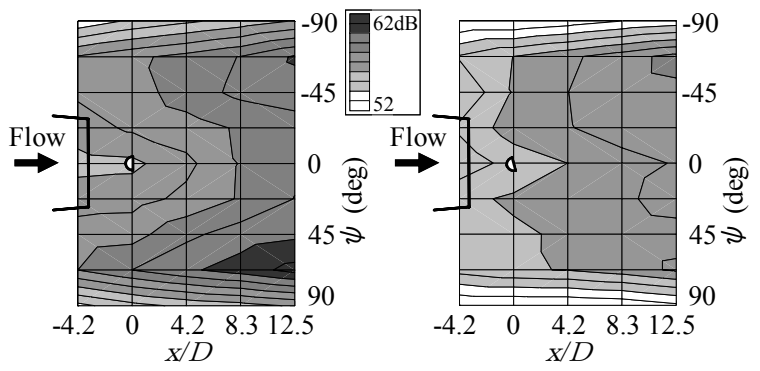

(a-1) $f_{\mathrm{m}}=4 \mathrm{kHz}, \quad \beta=0$ 。

(a-2) $f_{\mathrm{m}}=4 \mathrm{kHz}, \quad \beta=15$
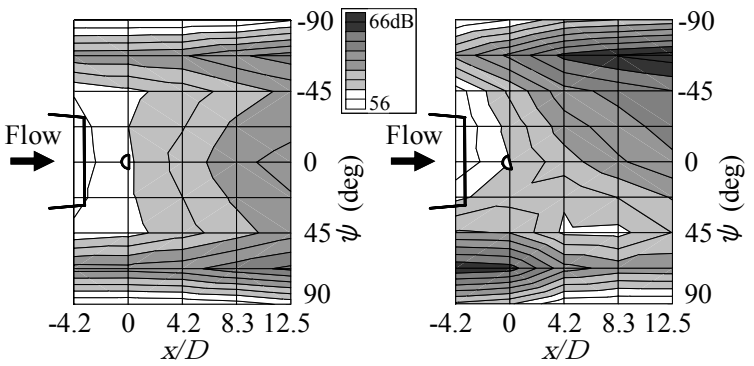

(b-1) $f_{\mathrm{m}}=1 \mathrm{kHz}, \quad \beta=0$

(b-2) $f_{\mathrm{m}}=1 \mathrm{kHz}, \quad \beta=15^{\circ}$

Fig. 19 1/1-octave, sound pressure level distribution $\left(R \mathrm{e}=1.9 \times 10^{5}\right)$

\section{Summary}

To obtain reliable experimental data that can be used for validation of a numerical method for predicting the aerodynamic sound generated by a bluff body, measurements were conducted using a simplified mock-up model of an automobile door mirror mounted on a flat plate and placed in a low-noise-level wind tunnel.

The large-scale flow structures around the model and the separation and transition of the boundary layer on the model surface were analyzed in detail using the measured time-averaged and fluctuating static pressure distributions on the model surface and flat plate. The streamlines on the model surface were visualized using an oil flow method. The frequency spectra of the sound pressure fluctuations and directivity of the radiated sound were also analyzed in conjunction with the flow-field characteristics. The major findings are summarized as follows.

(1) The pressure fluctuations on the model surface and flat plate indicated the existence of large-scale vortical motion with a non-dimensional frequency, estimated on the basis of the model width and the free-stream velocity, of 0.16 .

(2) The frequency spectra of the sound pressure fluctuations had a weak peak apparently due to the large-scale vortical motion. However, unlike the flow around a two-dimensional circular cylinder, the peak was not intense enough to alter the overall level of the sound radiated from the model.

(3) When the model was rotated 15 degrees, the power spectra of the sound pressure increased in the non-dimensional frequency range between 1 and 2 .

We greatly thank former graduate student Dr. Yasumasa Suzuki and technical staff member Mr. Stuneo Suzuki for their great help in obtaining reliable and accurate experimental data.

\section{References}

(1) K. Ono, et al., Prediction of wind noise radiated from passenger cars and its evaluation based on auralization, J. Wind Eng. Ind. Aerodyn., 81(1999) 403-419. 
(2) Y. Li, et al., Evaluation of Aerodynamic Noise Generated in Production Vehicle Using Experiment and Numerical Simulation, SAE Paper, 2003-01-1314 (2003).

(3) Y. Kato, Recent Advances in Numerical Analysis of Aeroacoustics, Recent Fluid Engineering for Better Automobile Performances, Proc. JSAE Symposium, 11-04 (2004) $54-55$.

(4) R. Hold, et al., Numerical Simulation of Aeroacoustic Sound Generated by Generic Bodies Placed on a Plate: Part 1-Prediction of Aeroacoustic Sources, AIAA Paper, 99-1896 (1999).

(5) R. Siegert, et al., Numerical Simulation of Aeroacoustic Sound Generated by Generic Bodies Placed on a Plate: Part 2-Prediction of Radiated Sound Pressure, AIAA Paper, 99-1895 (1999).

(6) Y. Sasaki, et al., Numerical Simulation of Aerodynamic Sound Radiated from a Blunted Body, JSAE Annual Congress Proceedings, 20-02 (2002) 9-12.

(7) H. Schlichting, Boundary-Layer Theory, Seventh Edition (1979) 16, McGraw-Hill.

(8) B. Cantwell and D. Coles, An Experimental Study of Entrainment and Transport in the Turbulent Near Wake of Circular Cylinder, J. Fluid Mech., 136 (1983), 321-374.

(9) The Japan Society of Mechanical Engineers, JSME Mechanical Engineers' Handbook, Fundamentals, A5 Fluids Engineering, (1986), A5-97, Maruzen. 